\title{
Cardiovascular magnetic resonance predicts all-cause mortality in pulmonary hypertension associated with heart failure with preserved ejection fraction
}

\author{
Pankaj Garg ${ }^{1,2} \circledast$. Robert A. Lewis ${ }^{1,2,3} \cdot$ Christopher S. Johns ${ }^{1,2} \cdot$ Andrew J. Swift $^{1,2}$. David Capener ${ }^{1}$. \\ Smitha Rajaram ${ }^{2}$ A. A. Roger Thompson ${ }^{1,2,3} \cdot$ Robin Condliffe $^{2,3} \cdot$ Charlie A. Elliot ${ }^{2,3}$. \\ Athanasios Charalampopoulos ${ }^{2,3} \cdot$ Abdul G. Hameed $^{2,3} \cdot$ Alexander Rothman $^{1,2,3} \cdot$ Jim M. Wild ${ }^{1} \cdot$ David G. Kiely $^{2,3}$
}

Received: 23 February 2021 / Accepted: 4 May 2021 / Published online: 12 May 2021

(c) The Author(s) 2021

\begin{abstract}
This study aimed to determine the prognostic value of cardiovascular magnetic resonance (CMR) in patients with heart failure with preserved ejection fraction and associated pulmonary hypertension (pulmonary hypertension-HFpEF). Patients with pulmonary hypertension-HFpEF were recruited from the ASPIRE registry and underwent right heart catheterisation (RHC) and CMR. On RHC, the inclusion criteria was a mean pulmonary artery pressure (MPAP) $\geq 25 \mathrm{mmHg}$ and pulmonary arterial wedge pressure $>15 \mathrm{mmHg}$ and, on CMR, a left atrial volume $>41 \mathrm{ml} / \mathrm{m}^{2}$ with left ventricular ejection fraction $>50 \%$. Cox regression was performed to evaluate CMR against all-cause mortality. In this study, 116 patients with pulmonary hypertension-HFpEF were identified. Over a mean follow-up period of $3 \pm 2$ years, 61 patients with pulmonary hypertension-HFpEF died (53\%). In univariate regression, 11 variables demonstrated association to mortality: indexed right ventricular (RV) volumes and stroke volume, right ventricular ejection fraction (RVEF), indexed RV mass, septal angle, pulmonary artery systolic/diastolic area and its relative area change. In multivariate regression, only three variables were independently associated with mortality: RVEF (HR 0.64, P <0.001), indexed RV mass (HR 1.46, P <0.001) and IV septal angle (HR 1.48, $\mathrm{P}<0.001)$. Our CMR model had 0.76 area under the curve $(\mathrm{P}<0.001)$ to predict mortality. This study confirms that pulmonary hypertension in patients with $\mathrm{HFpEF}$ is associated with a poor prognosis and we observe that CMR can risk stratify these patients and predict all-cause mortality. When patients with HFpEF develop pulmonary hypertension, CMR measures that reflect right ventricular afterload and function predict all-cause mortality.
\end{abstract}

Keywords Magnetic resonance imaging $\cdot$ Heart failure $\cdot$ Pulmonary hypertension $\cdot$ Prognosis $\cdot$ Right ventricular function

\section{Introduction}

Heart failure with preserved ejection fraction (HFpEF) now constitutes approximately half of all heart failure (HF) diagnoses [1]. It is estimated that approximately $50 \%$ of patients with HFpEF develop pulmonary hypertension $[2,3]$. The

Pankaj Garg

Pankaj.Garg@nhs.net

1 Department of Infection, Immunity and Cardiovascular Disease, University of Sheffield, Sheffield S102JF, England

2 Sheffield Teaching Hospitals NHS Foundation Trust, London, England

3 Sheffield Pulmonary Vascular Disease Unit, Royal Hallamshire Hospital, Sheffield S10 2JF, England development of pulmonary hypertension in HFpEF is mainly due to raised left ventricular filling pressures, which causes a sustained backward hemodynamic transmission to the pulmonary vascular bed. In patients with HFpEF, the development of pulmonary hypertension predicts a worse prognosis $[2,4]$.

Cardiovascular magnetic resonance (CMR) is the imaging reference standard for volumetric assessment and is emerging as one of the key non-invasive imaging methods to diagnose, and temporally monitor patients with pulmonary hypertension [5, 6] and phenotype patients with HFpEF [7]. The guidelines for the diagnosis and treatment of pulmonary hypertension highlight the potential of CMR in the assessment of disease severity and follow-up [8-10].

Cardiac MR provides added value to the clinical assessment in the prognostic evaluation of patients with pulmonary 
arterial hypertension [11] and allows for improved risk stratification of patients with PAH when used in conjunction with the REVEAL 2.0 and the modified French Pulmonary Hypertension Registry risk score [12]. In HFpEF, CMR can also sub-phenotype myocardial diseases that lead to the development of myocardial stiffness [13], including cardiac amyloidosis, hypertensive cardiomyopathy, hypertrophic cardiomyopathy and cardiac sarcoidosis.

Currently, there are limited outcome data in advanced stages of HFpEF using CMR, especially in patients with pulmonary hypertension-HFpEF. In addition, it remains unknown if CMR can be used in this patient population to risk stratify patients and predict prognosis [9].

The aim of this study was to investigate if non-contrast enhanced cine and flow CMR could risk stratify patients with pulmonary hypertension-HFpEF and inform prognosis.

\section{Methods}

This study was approved by the National Research Ethics Service (16/YH/0352) in the UK. The study complied with the Declaration of Helsinki. Patients were identified from the ASPIRE registry [14] and underwent a detailed assessment including blood, lung function, exercise testing, multimodality imaging and right heart catheterisation as previously described $[14,15]$. Consecutive patients with suspected pulmonary hypertension who underwent CMR from April 2012 to April 2017 were assessed for inclusion in the study. All patients were recruited at Sheffield Teaching Hospitals NHS Foundation Trust. Inclusion criteria included age $\geq 18$ years and a confirmed diagnosis of pulmonary hypertension-HFpEF. A diagnosis of pulmonary hypertension-HFpEF required a mean pulmonary arterial pressure $(\mathrm{mPAP}) \geq 25 \mathrm{mmHg}$ with a pulmonary artery wedge pressure (PCWP) $>15 \mathrm{mmHg}$ at right heart catheterisation (RHC) and a left ventricular ejection fraction $>50 \%$ with a left atrial volume index $>41 \mathrm{ml} / \mathrm{m}^{2}$ on CMR [16]. Right heart catheterisation was performed using a balloon-tipped 7.5Fr thermodilution catheter (Becton-Dickinson, Franklin Lakes, New Jersey). The mean pulmonary arterial pressure and pulmonary artery wedge pressure were recorded using standard techniques described previously [17]. Patients with other forms of pulmonary hypertension were excluded.

\section{CMR acquisition}

CMR was performed on a GE HDx 1.5-T system (GE Healthcare, Milwaukee, Wisconsin) using an 8-channel cardiac coil.

The protocol included four-chamber (4Ch) and shortaxis (SA) cine images, acquired using a retrospectively cardiac gated multi-slice steady-state free precession (SSFP) sequence. We acquired a stack of axial images in the short axis (SA) plane, with a slice thickness of $10 \mathrm{~mm}$ with no inter-slice gap or $8 \mathrm{~mm}$ with a $2 \mathrm{~mm}$ inter-slice gap, from the base to the apex of both ventricles. Time-resolved images of the pulmonary artery were performed using a retrospectively cardiac gated SSFP sequence with a single slice of $10 \mathrm{~mm}$ taken perpendicular to the long-axis of the pulmonary artery. The SSFP sequence parameters were: TR $2.8 \mathrm{~ms}$, TE $1.0 \mathrm{~ms}$, flip angle $50^{\circ}$, field of view $48 \times 48,256 \times 256$ matrix and $125 \mathrm{kHz}$ bandwidth.

\section{CMR image analysis}

CMR images were manually analysed on GE Advantage Workstation ReportCard software, by an experienced radiographer (DC). All segmentation was done manually. Ventricular volumetric assessment was performed as per guidelines [18] (S-Fig. 1-2). All volume parameters were indexed to body surface area. Metrics included: the indexed left ventricular end-diastolic volume (LVEDVi), end-systolic volume (LVESVi), stroke volume (LVSVi), left ventricular ejection fraction (LVEF), right ventricular end-diastolic volume (RVEDVi), end-systolic volume (RVESVi), stroke volume (RVSVi) and right ventricular ejection fraction (RVEF). Left atrial volume indexed was calculated using the biplane area-length method [19]. The interventricular (IV) septal angle were measured as previously described [20]. Maximal and minimal pulmonary arterial (PA) areas were manually traced, and relative area change (RAC) was defined by the following equation: PA RAC: (maximum area-minimum area) /minimum area [21]. Reproducibility for these CMR metrics have been previously published by our group [11, 20].

\section{Statistics}

Statistical analysis was performed using SPSS statistics 22 (IBM, Chicago). All continuous variables are presented as mean (standard deviation). Independent T-test was used to compare the clinical and CMR variables in alive/dead patients. For categorical comparisons, the Chi-square test was used. Further details on statistical analysis are in the supplementary online file. A P-value of 0.05 was considered statistically significant.

\section{Results}

\section{Study population}

The baseline demographics of the 116 patients with pulmonary hypertension-HFpEF who met the inclusion criteria are shown in Table 1 . The average age was $73 \pm 7$ years and $57 \%$ 
Table 1 Study demographics and pulmonary haemodynamics

\begin{tabular}{llllr}
\hline & All & Alive & Dead & P-value* \\
\hline $\mathrm{N}$ & 116 & 55 & 61 & \\
Age (yrs) & $73 \pm 7$ & $71 \pm 8$ & $75 \pm 7$ & 0.018 \\
Gender (Male) & $50(43 \%)$ & $15(27 \%)$ & $35(57 \%)$ & 0.001 \\
BMI (kg/m $\left.{ }^{2}\right)$ & $31 \pm 6$ & $31 \pm 7$ & $30 \pm 6$ & 0.284 \\
Heart rate (bpm) & $65 \pm 12$ & $65 \pm 13$ & $65 \pm 11$ & 0.800 \\
Diabetes mellitus & $30(26 \%)$ & $13(24 \%)$ & $17(28 \%)$ & 0.607 \\
Hypercholesterol- & $24(21 \%)$ & $11(20 \%)$ & $13(21 \%)$ & 0.863 \\
aemia & & & & \\
Hypertension & $78(67 \%)$ & $41(75 \%)$ & $37(61 \%)$ & 0.056 \\
IHD & $20(17 \%)$ & $9(16 \%)$ & $11(18 \%)$ & 0.814 \\
Atrial fibrillation & $82(71 \%)$ & $32(58 \%)$ & $50(82 \%)$ & 0.009 \\
Stroke & $8(7 \%)$ & $3(5 \%)$ & $5(8 \%)$ & 0.565 \\
Invasive haemodynamics & & & \\
Mean RA (mmHg) & $15 \pm 5$ & $14 \pm 5$ & $17 \pm 5$ & 0.004 \\
Mean PAP (mmHg) & $42 \pm 10$ & $39 \pm 9$ & $45 \pm 10$ & 0.002 \\
PCWP (mmHg) & $23 \pm 5$ & $23 \pm 5$ & $22 \pm 5$ & 0.327 \\
DPG (mmHg) & $1 \pm 7$ & $-1 \pm 6$ & $3 \pm 7$ & $<0.001$ \\
TPG (mmHg) & $19 \pm 9$ & $16 \pm 7$ & $22 \pm 9$ & $<0.001$ \\
Cardiac index (L/ & $2.8 \pm 1$ & $2.9 \pm 1.1$ & $2.7 \pm 0.8$ & 0.432 \\
$\quad$ min/m $\left.{ }^{2}\right)$ & & & & \\
PVR (Wood unit) & $4.2 \pm 2.95$ & $3.16 \pm 1.7$ & $5 \pm 3.5$ & $<0.001$ \\
O2 saturation (arte- & $95 \pm 4$ & $95 \pm 3$ & $94 \pm 4$ & 0.071 \\
$\quad$ rial) & & & & \\
O2 saturation & $65 \pm 9$ & $68 \pm 8$ & $63 \pm 10$ & 0.005 \\
$\quad$ (venous) & & & & \\
\hline
\end{tabular}

Categorical comparisons done by Chi-square test

$B M I$ Body mass index, $I H D$ Ischaemic heart disease, $R A$ right atrial, $P A P$ pulmonary artery pressure, $P C W P$ pulmonary capillary wedge pressure, $D P G$ diastolic pulmonary gradient, $T P G$ transpulmonary pressure gradient, $P V R$ pulmonary vascular resistance

* P-value is for T-Test comparison between Alive and Dead cohorts

of patients were female. During the mean follow-up period of $3 \pm 2$ years, 61 patients with pulmonary hypertensionHFpEF died (53\%).

\section{Left heart volumetric assessment}

Left atrial volume index was significantly higher in the patients who were dead at the census $\left(80.3 \pm 31.2 \mathrm{ml} / \mathrm{m}^{2}\right.$ vs $66.5 \pm 19.1 \mathrm{ml} / \mathrm{m}^{2}, \mathrm{P}=0.005$ ), whereas, there were no significant differences in LV volumes (LVEDVi, LVESVi), LV systolic function assessed by LVEF, LV stroke volume index or LV mass (indexed) (Supplementary Table 1).

\section{Right heart volumetric assessment}

Right ventricular volumetric parameters including RVESVi, RV mass and interventricular septal angle were significantly higher $(\mathrm{P}<0.005)$ and RVEF significantly lower $(\mathrm{P}=0.002)$ in patients with pulmonary hypertension-HFpEF who were dead compared to those alive at the census date (Fig. 1A).

\section{Pulmonary artery area}

The PA diastolic area was significantly increased $(\mathrm{P}=0.003)$. PA systolic area was increased $(\mathrm{P}=0.016)$ and $\mathrm{PA}$ relative area change reduced $(\mathrm{P}=0.027)$ in patients who were dead compared to those alive at the census data.

At the univariate analysis of demographic and CMR variables, 11 were associated with mortality (Supplementary Table 2). In a forward selection, multivariate cox regression model, only three parameters demonstrated independent association to all-cause mortality in this patient population. These included the CMR parameters-RVEF (HR 0.64, 95\% CI 0.47 to $0.87, \mathrm{P}<0.001$ ), indexed RV mass (HR $1.46,95 \%$ CI 1.18 to $1.8, \mathrm{P}<0.001)$ and IV septal angle (HR $1.48,95 \%$ CI 1.12 to $1.94, \mathrm{P}<0.001$ ) (Fig. 1).

Pulmonary hypertension-HFpEF with RVEF less than or equal to $49 \%$ had a worse survival at 1 -year $(70 \%$ vs $96 \%$ ), 3-years (44\% vs $73 \%)$ and 5 -years (26\% vs $64 \%)$, than patients with a RVEF $>49 \%$, respectively $(\mathrm{P}=0.0001)$ (Fig. 2). In patients with an indexed RV mass $>17 \mathrm{~g} / \mathrm{m}^{2}$ patients had a worse survival at 1-year (71\% vs. $93 \%)$, 3 -years (39\% vs. $76 \%)$ and at 5 -years $(30 \%$ vs. $60 \%)$ than patients with indexed $\mathrm{RV}$ mass $<=17 \mathrm{~g} / \mathrm{m}^{2}(\mathrm{P}=0.0002)$. Patients with an inter-ventricular septal angle $>139^{\circ}$ had a worse survival at 1 -year (71\% vs. $90 \%), 3$-years $(31 \%$ vs. $74 \%)$ and at 5 -years (25\% vs. $54 \%)$ than patients with an inter-ventricular septal angle $<=139^{\circ}(\mathrm{P}=0.0001)$. Receiver operating characteristic analysis are detailed in Table 2 and the supplementary document.

A score was developed defined by thresholds from receiver operator characteristic (ROC) analysis. The minimum score was set at 0 which meant all the three variables were not above their threshold. The maximum score was 3 which meant all the three variables were above their threshold. The CMR score model demonstrated a higher area under the curve than the three variables individually at $0.76,95 \%$ CI 0.67 to $0.83, \mathrm{P}<0.001$. The overall sensitivity, specificity and accuracy of a pulmonary hypertension-HFpEF CMR score $>2$ for predicting all-cause mortality were $72 \%(95 \%$ CI 59.2-82.9), 71\% (95\% CI 57.1-82.4) and 71.5\% (95\% CI 62.4-79.5) respectively (Fig. 2).

\section{Discussion}

In this study we have shown that a CMR based volumetric and functional assessment of the right ventricle can risk stratify patients with pulmonary hypertension-HFpEF. In addition, we have presented a simple CMR scoring model incorporating independent predictors of outcome RV EF, 

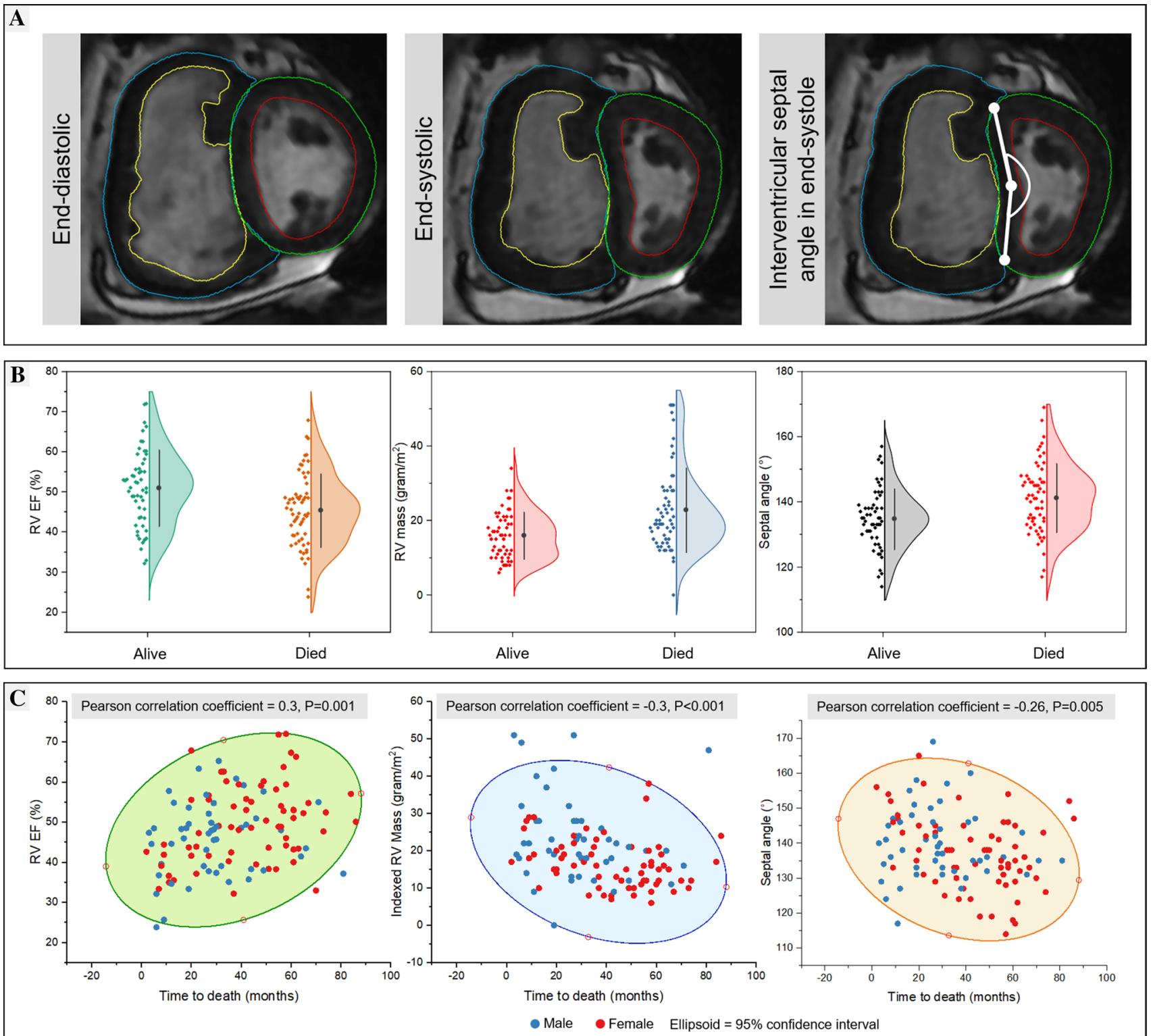

Fig. 1 Panel a Illustration of study case. There is RV hypertrophy with reduced RV function (RV EF 40\%) and the septal angle is $190^{\circ}$. Panel b Mean plots for RV EF, indexed mass and septal angle. Panel c Scatter plots for the three variables against time-to-death

indexed RV mass and IV septal angle. Our study highlights the potential value of CMR in the prognostic assessment of patients with pulmonary hypertension-HFpEF.

Echocardiography is a first-line diagnostic imaging test for patients presenting with symptoms and signs of HF [22]. Several studies have demonstrated that echocardiographic measures reflecting RV function predict prognosis in pulmonary hypertension-HFpEF [2, 23-25]. It is worth noting that the majority of echocardiographybased studies have demonstrated that it is mainly RV function and remodelling that are associated with a poor prognosis in pulmonary hypertension-HFpEF. In a study which recruited 419 patients with HFpEF, Burke et al. demonstrated that LV compliance and also RV hypertrophy (hazard ratio $=1.37 ; \mathrm{P}<0.001$ ), were most predictive of worse outcomes [24]. Similar to our study, neither LV function nor any other LV volumetric parameter was independently associated with cardiovascular outcomes. In another large study of 562 patients which assessed $\mathrm{RV}$ function in a semiquantitative way, Mohammed et al. demonstrated that the presence of impaired RV function was associated with higher all-cause mortality (hazard ratio $=1.35 ; \mathrm{P}=0.03$ ) [23]. It is worth noting that even though echocardiography is a good screening imaging modality, evaluation of right heart remains challenging and limited. Moreover, almost $10-15 \%$ patients can have 


\section{A}

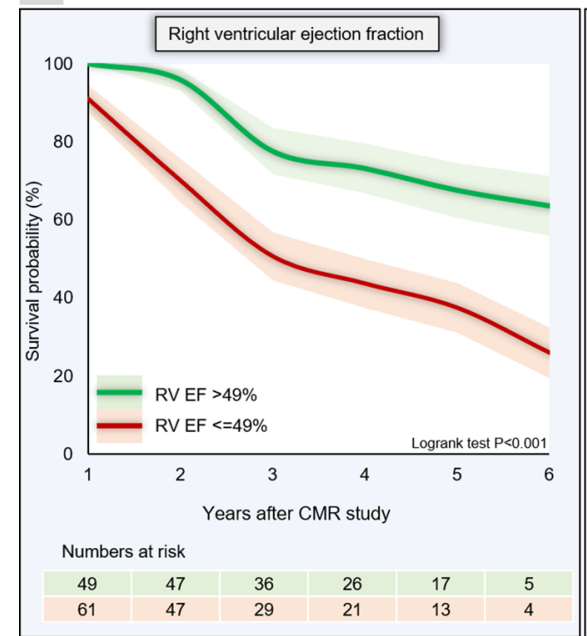

B 6

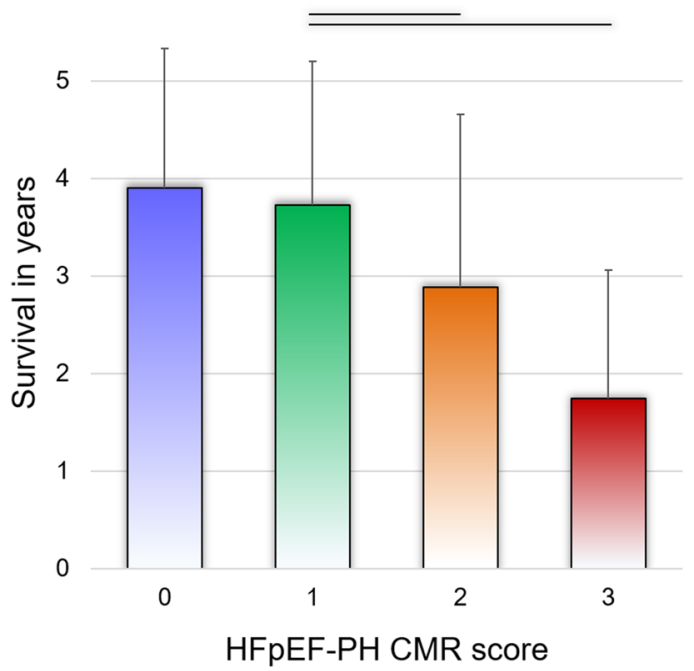

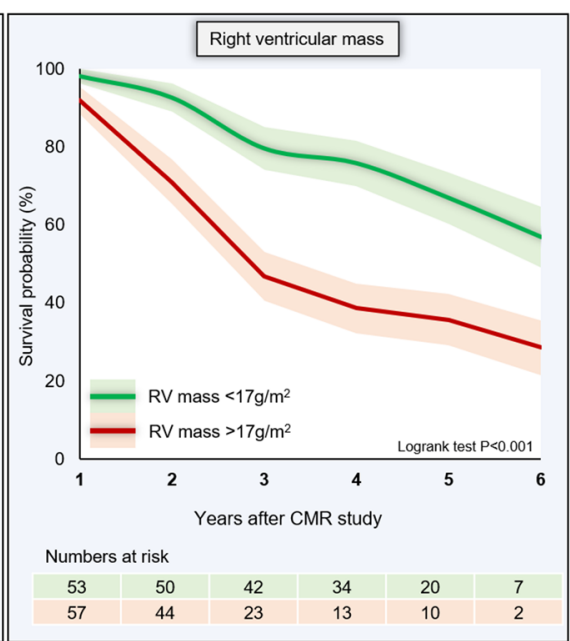

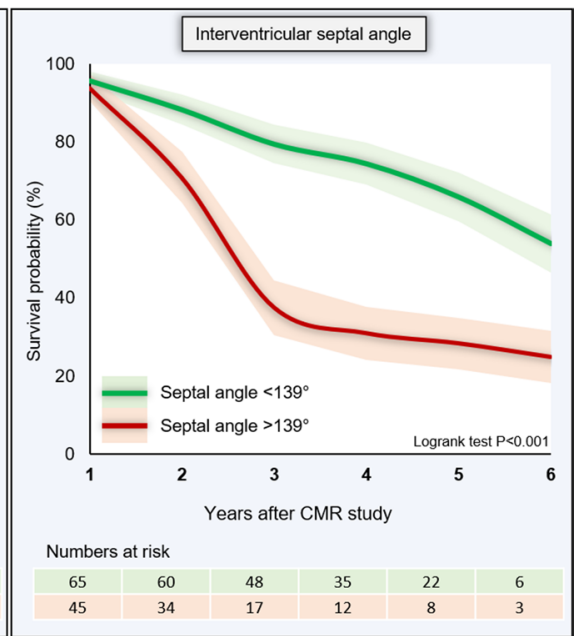

C

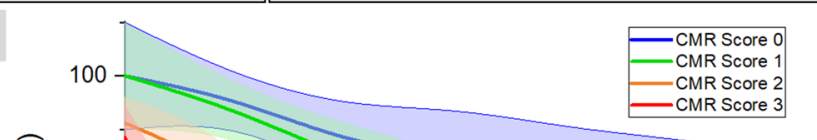

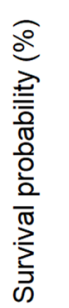
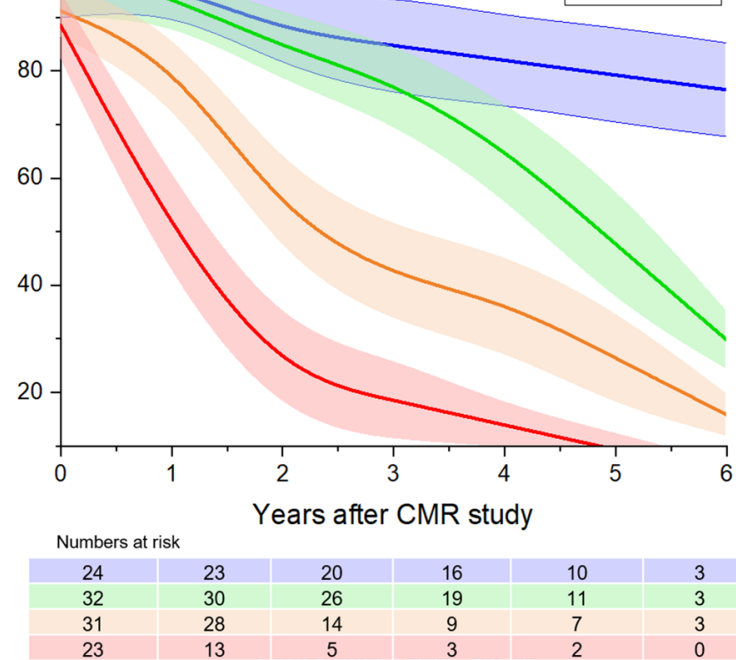

Fig. 2 Panel aKaplan-Meier survival curves. Panel b CMR score model predicts survival in pulmonary hypertension-HFpEF. Panel c KaplanMeier survival curve results for the HFpEF-PH CMR score

Table 2 C-statistics

\begin{tabular}{lllllll}
\hline & AUC & $95 \%$ CI & P & Criterion & Sensitivity (95\% CI) & Specificity (95\% CI) \\
\hline RV EF (\%) & $0.67^{\text {a }}$ & 0.57 to 0.75 & $<0.01$ & $\leq 49$ & $75(63-85.5)$ & $58(44-71)$ \\
Indexed RV mass $\left(\right.$ grams $\left./ \mathrm{m}^{2}\right)$ & 0.70 & 0.61 to 0.78 & $<0.01$ & $>17$ & $67(54-79)$ & $62(48-75)$ \\
IV septal angle $\left(^{\circ}\right)$ & $0.67^{\text {a }}$ & 0.58 to 0.76 & $<0.01$ & $>139$ & $57(44-70)$ & $76(63-87)$ \\
CMR model & 0.76 & 0.67 to 0.83 & $<0.01$ & $>1$ & $72(59-83)$ & $71(57-82)$ \\
\hline
\end{tabular}

$A U C$ area under the curve, $C I$ confidence interval, $R V E F$ Right ventricular ejection fraction, $I V$ intra-ventricular, $C M R$ cardiac magnetic resonance

${ }^{\text {a }}$ Significantly different to CMR model AUC

${ }^{\mathrm{b}}$ Youden index derived

non-diagnostic studies due to poor acoustic windows. The role of CMR is not only to clarify the diagnosis, but also monitor disease process longitudinally. CMR uniquely allows one to quantify RV mass precisely, which cannot 
be done by echocardiography. Hence, CMR is the reference standard for the evaluation of right cardiac volumes and function [26].

It is established that RV dysfunction on CMR predicts outcomes in pulmonary arterial hypertension [27]. The findings of this study are broadly consistent with Aschauer et al. who also demonstrated that right ventricular systolic dysfunction by CMR is independently associated with mortality in HFpEF [28]. However, the main differences between their work and this study are that they recruited generic HFpEF patients where as in this study we only recruited patients who had developed PH-HFpEF. Hence, it is plausible to conclude that this study has recruited more severe cases of HFpEF, who have subsequently developed PH. In addition, this study has evaluated RV functional and anatomy comprehensively including septal angle which demonstrated independent prognostic role in patients with $\mathrm{PH}-\mathrm{HFpEF}$.

In this study, we have also developed a simplified scoring tool based on three CMR metrics which may aid risk stratification of patients with pulmonary hypertension-HFpEF. The CMR score is simple and easy to integrate in routine practice. Patients with CMR score $>2$, could be offered more regular clinical monitoring as evidence suggests that a strategy to reduce the pulmonary artery pressure primarily by diuretic therapy can improve outcomes in these patients [29, 30]. Hence, a non-invasive CMR model which can appropriately risk stratify patients by assessing right ventricular function and the severity of pre-capillary pulmonary hypertension may be of value in clinical trials of new therapies or treatment approaches including closer monitoring and optimisation of heart failure in high-risk patients.

\section{Limitations}

Patients were required to have a cardiac catheterisation to identify patients with HFpEF with pulmonary hypertension. The results of our study, therefore, apply to a selected cohort, however, this has the advantage of using reference standard haemodynamic measures to define pulmonary hypertension-HFpEF. HFpEF patients with a detectable scar, fibrosis on T1-mapping, myocardial ischaemia or right ventricular impairment appear to have a worse prognosis [31, 32]. In our study, we only evaluated CMR cine related parameters. Further studies are needed to evaluate prognostic role of multi-parametric mapping in pulmonary hypertension-HFpEF. However, we feel the current CMR model has value in patients who either have contraindication for gadolinium contrast agent or are due to have a shorter CMR scan for monitoring of function. Finally, this study did not record echocardiography data to evaluate incremental role of CMR.

\section{Conclusion}

In this study, we observed that CMR can risk stratify pulmonary hypertension-HFpEF patients and predict all-cause mortality. When patients develop pulmonary hypertension associated with $\mathrm{HFpEF}$, it is primarily right heart function and imaging features of pre-capillary pulmonary hypertension which predict mortality.

Supplementary Information The online version contains supplementary material available at https://doi.org/10.1007/s10554-021-02279-z.

Acknowledgements We thank the MRI staff at the University of Sheffield for peforming CMR scans.

Author contributions Conceptualization: All authors; data curation: PG, CSJ, AJS, RAL; formal analysis: RAL, PG; acquisition: PG, JMW and AJS; investigation: all authors; methodology: all authors; project administration: AJS and DGK; resources: DGK and JMW; writing: all authors.

Funding This work was partly funded by the Wellcome Trust (205188/Z/16/Z), British Heart Foundation Grant to AART (FS/18/13/33281) and MRC Grant MR/M008894/1.

\section{Declarations}

Conflict of interest PG has advisory role to Pie Medical Imaging and Medis Medical Imaging.

Open Access This article is licensed under a Creative Commons Attribution 4.0 International License, which permits use, sharing, adaptation, distribution and reproduction in any medium or format, as long as you give appropriate credit to the original author(s) and the source, provide a link to the Creative Commons licence, and indicate if changes were made. The images or other third party material in this article are included in the article's Creative Commons licence, unless indicated otherwise in a credit line to the material. If material is not included in the article's Creative Commons licence and your intended use is not permitted by statutory regulation or exceeds the permitted use, you will need to obtain permission directly from the copyright holder. To view a copy of this licence, visit http://creativecommons.org/licenses/by/4.0/.

\section{References}

1. Dunlay SM, Roger VL, Redfield MM (2017) Epidemiology of heart failure with preserved ejection fraction. Nat Rev Cardiol 14(10):591-602

2. Lam CSP, Roger VL, Rodeheffer RJ, Borlaug BA, Enders FT, Redfield MM (2009) Pulmonary hypertension in heart failure with preserved ejection fraction: a community-based study. J Am Coll Cardiol 53(13):1119-1126

3. Rosenkranz S, Gibbs JSR, Wachter R, De Marco T, Vonk-Noordegraaf A, Vachiéry JL (2016) Left ventricular heart failure and pulmonary hypertension. Eur Heart J 37(12):942-954

4. Shah SJ (2013) Matchmaking for the optimization of clinical trials of heart failure with preserved ejection fraction: no laughing matter. J Am Coll Cardiol 62(15):1339-1342 
5. Bradlow WM, Gibbs JSR, Mohiaddin RH (2012) Cardiovascular magnetic resonance in pulmonary hypertension. J Cardiovasc Magn Reson 14(1):6

6. Kiely DG, Levin DL, Hassoun PM, Ivy D, Jone P-N, Bwika J et al (2019) Statement on imaging and pulmonary hypertension from the Pulmonary Vascular Research Institute (PVRI). Pulm Circ 9(3):204589401984199

7. Chamsi-Pasha MA, Zhan Y, Debs D, Shah DJ (2019) CMR in the evaluation of diastolic dysfunction and phenotyping of HFpEF: current role and future perspectives. JACC Cardiovasc Imaging 13(1):283-296

8. Galiè N, Humbert M, Vachiery JL, Gibbs S, Lang I, Torbicki A et al (2016) 2015 ESC/ERS guidelines for the diagnosis and treatment of pulmonary hypertension. Eur Heart J 37(1):67-119

9. Peacock AJ, Crawley S, McLure L, Blyth KG, Vizza CD, Poscia $R$ et al (2014) Changes in right ventricular function measured by cardiac magnetic resonance imaging in patients receiving pulmonary arterial hypertension-targeted therapy: the EURO-MR study. Circ Cardiovasc Imaging 7(1):107-114

10. Hassoun PM, Zamanian RT, Damico R, Lechtzin N, Khair R, Kolb $\mathrm{TM}$ et al (2015) Ambrisentan and tadalafil up-front combination therapy in scleroderma-associated pulmonary arterial hypertension. Am J Respir Crit Care Med 192(9):1102-1110

11. Swift AJ, Capener D, Johns C, Hamilton N, Rothman A, Elliot C et al (2017) Magnetic resonance imaging in the prognostic evaluation of patients with pulmonary arterial hypertension. Am J Respir Crit Care Med 196(2):228-39

12. Lewis RA, Johns CS, Cogliano M, Capener D, Tubman E, Elliot CA et al (2019) Identification of cardiac MRI thresholds for risk stratification in pulmonary arterial hypertension. Am J Respir Crit Care Med. https://doi.org/10.1164/rccm.201909-1771OC

13. Kanagala P, Cheng ASH, Singh A, McAdam J, Marsh AM, Arnold JR et al (2018) Diagnostic and prognostic utility of cardiovascular magnetic resonance imaging in heart failure with preserved ejection fraction-implications for clinical trials. J Cardiovasc Magn Reson 20(1):4

14. Hurdman J, Condliffe R, Elliot CA, Davies C, Hill C, Wild JM et al (2012) ASPIRE registry: assessing the spectrum of pulmonary hypertension identified at a REferral centre. Eur Respir J 39(4):945-955

15. Swift AJ, Rajaram S, Capener D, Elliot C, Condliffe R, Wild JM et al (2015) Longitudinal and transverse right ventricular function in pulmonary hypertension: cardiovascular magnetic resonance imaging study from the ASPIRE registry. Pulm Circ 5(3):557-564

16. Crawley SF, Johnson MK, Dargie HJ, Peacock AJ (2013) LA volume by CMR distinguishes idiopathic from pulmonary hypertension due to HFpEF. JACC Cardiovasc Imaging 6(10):1120-1121

17. Swift AJ, Rajaram S, Hurdman J, Hill C, Davies C, Sproson TW et al (2013) Noninvasive estimation of PA pressure, flow, and resistance with CMR imaging. JACC Cardiovasc Imaging 6(10):1036-1047

18. Kramer CM, Barkhausen J, Flamm SD, Kim RJ, Nagel E (2008) Standardized cardiovascular magnetic resonance imaging (CMR) protocols, society for cardiovascular magnetic resonance: board of trustees task force on standardized protocols. J Cardiovasc Magn Reson 10(1):35

19. Khan MA, Yang EY, Zhan Y, Judd RM, Chan W, Nabi F et al (2019) Association of left atrial volume index and all-cause mortality in patients referred for routine cardiovascular magnetic resonance: a multicenter study. J Cardiovasc Magn Reson. https://doi. org/10.1186/s12968-018-0517-0
20. Johns CS, Kiely DG, Rajaram S, Hill C, Thomas S, Karunasaagarar K et al (2018) Diagnosis of pulmonary hypertension with cardiac MRI: derivation and validation of regression models. Radiology 290(1):61-68

21. Swift AJ, Rajaram S, Condliffe R, Capener D, Hurdman J, Elliot $\mathrm{C}$ et al (2012) Pulmonary artery relative area change detects mild elevations in pulmonary vascular resistance and predicts adverse outcome in pulmonary hypertension. Invest Radiol 47(10):571-577

22. Ponikowski P, Voors AA, Anker SD, Bueno H, Cleland JGF, Coats AJS et al (2016) ESC guidelines for the diagnosis and treatment of acute and chronic heart failure. Eur Heart J 37(27):2129-2200

23. Mohammed SF, Hussain I, AbouEzzeddine OF, Abou Ezzeddine OF, Takahama H, Kwon SH et al (2014) Right ventricular function in heart failure with preserved ejection fraction: a community-based study. Circulation 130(25):2310-2320

24. Burke MA, Katz DH, Beussink L, Selvaraj S, Gupta DK, Fox J et al (2014) Prognostic importance of pathophysiologic markers in patients with heart failure and preserved ejection fraction. Circ Heart Fail 7(2):288-299

25. Goliasch G, Zotter-Tufaro C, Aschauer S, Duca F, Koell B, Kammerlander AA et al (2015) Outcome in heart failure with preserved ejection fraction: the role of myocardial structure and right ventricular performance. PLOS ONE 10(7):e0134479

26. Grothues F, Moon JC, Bellenger NG, Smith GS, Klein HU, Pennell DJ (2004) Interstudy reproducibility of right ventricular volumes, function, and mass with cardiovascular magnetic resonance. Am Heart J 147(2):218-223

27. Baggen VJM, Leiner T, Post MC, van Dijk AP, Roos-Hesselink JW, Boersma E et al (2016) Cardiac magnetic resonance findings predicting mortality in patients with pulmonary arterial hypertension: a systematic review and meta-analysis. Eur Radiol 26(11):3771-3780

28. Aschauer S, Kammerlander AA, Zotter-Tufaro C, Ristl R, Pfaffenberger S, Bachmann A et al (2016) The right heart in heart failure with preserved ejection fraction: insights from cardiac magnetic resonance imaging and invasive haemodynamics. Eur J Heart Fail 18(1):71-80

29. Abraham WT, Stevenson LW, Bourge RC, Lindenfeld JA, Bauman JG, Adamson PB et al (2016) Sustained efficacy of pulmonary artery pressure to guide adjustment of chronic heart failure therapy: complete follow-up results from the CHAMPION randomised trial. Lancet Lond Engl 387(10017):453-461

30. Abraham WT, Adamson PB, Bourge RC, Aaron MF, Costanzo MR, Stevenson LW et al (2011) Wireless pulmonary artery haemodynamic monitoring in chronic heart failure: a randomised controlled trial. Lancet Lond Engl 377(9766):658-666

31. Assadi H, Jones R, Swift AJ, Al-Mohammad A, Garg P (2021) Cardiac MRI for the prognostication of heart failure with preserved ejection fraction: a systematic review and meta-analysis. Magn Reson Imaging 76:116-122

32. Garg P, Assadi H, Jones R, Chan WB, Metherall P, Thomas R et al (2021) Left ventricular fibrosis and hypertrophy are associated with mortality in heart failure with preserved ejection fraction. Sci Rep 11(1):617

Publisher's Note Springer Nature remains neutral with regard to jurisdictional claims in published maps and institutional affiliations. 